\title{
Ascending aorta dilation in association with bicuspid aortic valve: A maturation defect of the aortic wall
}

\author{
Nimrat Grewal, MD, ${ }^{\text {a,b }}$ Adriana C. Gittenberger-de Groot, PhD, ${ }^{\text {b,c }}$ Robert E. Poelmann, PhD, \\ Robert J. M. Klautz, MD, PhD, ${ }^{a}$ Johannes H. N. Lindeman, MD, PhD, ${ }^{\mathrm{d}}$ Marie-José Goumans, PhD, \\ Meindert Palmen, MD, PhD, ${ }^{\mathrm{a}}$ Salah A. Mohamed, PhD, ${ }^{\mathrm{f}}$ Hans-Hinrich Sievers, $\mathrm{MD},{ }^{\mathrm{f}}$ \\ Ad J. J. C. Bogers, MD, PhD, ${ }^{g}$ and Marco C. DeRuiter, $\mathrm{PhD}^{\mathrm{b}}$
}

\begin{abstract}
Objective: Patients with a bicuspid aortic valve have increased susceptibility to the development of ascending aortic dilation and dissection compared with persons with a tricuspid valve. To unravel a possible different mechanism underlying dilation in bicuspidy and tricuspidy, a comparison of the structure of the aortic wall was made.
\end{abstract}

\begin{abstract}
Methods: Ascending aortic wall biopsies were divided into 4 groups: bicuspid $(n=36)$ and tricuspid $(n=23)$ without and with dilation. The expression of vascular smooth muscle cell maturation markers including lamin $\mathrm{A} / \mathrm{C}$, which plays a pivotal role in smooth muscle cell differentiation, and its splicing variant progerin indicative of aging, were studied immunohistochemically. Attention was also paid to the inflammatory status.
\end{abstract}

\begin{abstract}
Results: There is a significant difference in the structure and maturation of the aortic wall in bicuspidy, persisting in the dilated aortic wall, presenting with a thinner intima, lower expression of $\alpha$ smooth muscle actin, smooth muscle $22 \alpha$, calponin, and almost absent expression of smoothelin. We show for the first time significantly lowered lamin A/C expression in bicuspidy. Progerin was found to be significantly increased in the media of the dilated wall in tricuspidy, also showing increased periaortic inflammation.
\end{abstract}

Conclusions: The structure of the nondilated and dilated aortic wall in bicuspidy and tricuspidy are intrinsically different, with the latter having more aspects of aging. In bicuspidy there is a defective smooth muscle cell differentiation possibly linked to lowered lamin $\mathrm{A} / \mathrm{C}$ expression. Based on this vessel wall immaturity and increased susceptibility to dilation, different diagnostic and therapeutic approaches are warranted. (J Thorac Cardiovasc Surg 2014;148:1583-90)

\begin{abstract}
A bicuspid aortic valve (BAV) is characterized by an aortic valve with 2 semilunar leaflets. BAV is the most common congenital cardiovascular malformation with a prevalence of $0.5 \%$ to $2 \%{ }^{1}$ The incidence of thoracic aortic dilation or aneurysm formation and dissection in patients with BAV is considered to be $50 \%$ to $70 \%{ }^{2}$ Compared with patients with a tricuspid aortic valve (TAV), patients with BAV have larger aortic root dimensions and a higher progression rate of dilation, ${ }^{3,4}$ suggesting that the process of dilation of the thoracic aorta is different in BAV compared with TAV. The terms aneurysm and dilation for

\footnotetext{
From the Departments of Cardiothoracic Surgery, ${ }^{\mathrm{a}}$ Anatomy and Embryology, ${ }^{\mathrm{b}}$ Cardiology, ${ }^{\mathrm{c}}$ Vascular Surgery, ${ }^{\mathrm{d}}$ Molecular Cell Biology, ${ }^{\mathrm{e}}$ Leiden University Medical Center, Leiden, The Netherlands; Department of Cardiac and Thoracic Vascular Surgery, ${ }^{\mathrm{f}}$ University of Lübeck, Lübeck, Germany; and Department of Cardiothoracic Surgery and Heart Valve Bank, ${ }^{\mathrm{g}}$ Erasmus University Medical Center, Rotterdam, The Netherlands.

Disclosures: J.H.N. Lindeman has acted as a consultant for Cardoz and Eli Lilly.

All other authors have nothing to disclose with regard to commercial support.

Received for publication Sept 6, 2013; revisions received Jan 6, 2014; accepted for publication Jan 21, 2014; available ahead of print Feb 19, 2014.

Address for reprints: Marco C. DeRuiter, PhD, Department of Anatomy and Embryology, Leiden University Medical Center, Postal zone: S-5-24, PO Box 9600, 2300 RC Leiden, The Netherlands (E-mail: m.c.deruiter@lumc.nl). $0022-5223 / \$ 36.00$

Copyright (c) 2014 by The American Association for Thoracic Surgery http://dx.doi.org/10.1016/j.jtcvs.2014.01.027
}

the aortopathy are used interchangeably. We have chosen to use the term dilation for clarity. Turbulent flow, as a result of asymmetric movement of valve leaflets in BAV, has been postulated as an essential determinant for the development of aortic dilation. ${ }^{5}$ An alternative hypothesis, that aortic dilation in BAV is mainly based on the intrinsic structure of the aortic wall, ${ }^{6}$ is supported by a high incidence of dilation in asymptomatic patients with BAV as well as dilation observed after aortic valve replacement. ${ }^{7}$ The latter hypothesis is further supported by reported altered molecular and/or metabolic characteristics in the aortic wall and valve leaflets in BAV, differences in elastic lamellae, loose attachment of vascular smooth muscle cells (VSMCs) to their surrounding elastic lamellae, and precocious VSMC apoptosis. ${ }^{8-17}$ Most studies have focused on the differences between the dilated TAV and BAV wall. The exact mechanisms underlying the development and progression of an aorta with normal dimensions into dilation in patients with TAV versus BAV, however, have not been delineated. In this study, we used a unique opportunity to compare nondilated ascending aortic wall specimens from patients with BAV, representative of a specific architecture and possible early lesions rather than of end-stage disease, with the normal aortic wall in TAV. 


$$
\begin{aligned}
& \text { Abbreviations and Acronyms } \\
& \alpha \text { SMA }=\alpha \text { smooth muscle actin } \\
& \text { BA }=\text { bicuspid aortic valve without dilation } \\
& \text { BAD }=\text { bicuspid aortic valve with dilation } \\
& \text { BAV } \text { bicuspid aortic valve } \\
& \text { BSA } \text { bovine serum albumin } \\
& \text { DAB }=\text { diaminobenzidine tetrachloride } \\
& \text { GAPDH }=\text { glyceraldehyde-3-phosphate } \\
& \text { dehydrogenase } \\
& \text { LUMC }=\text { Leiden University Medical Center } \\
& \text { MF }=\text { microscopic field } \\
& \text { PBS }=\text { phosphate buffered saline } \\
& \text { PBS-T }=\text { phosphate buffered saline with } 0.05 \% \\
& \text { SM22 } \text { Tween-20 } \\
& \text { TA }=\text { tricuspid aortic valve without dilation } \\
& \text { TAD }=\text { tricuspid aortic valve with dilation } \\
& \text { TAV }=\text { tricuspid aortic valve } \\
& \text { VSMC }=\text { vascular smooth muscle cell }
\end{aligned}
$$

Furthermore, these data could be compared with the histopathology of the dilated vessel wall in BAV and TAV. Histologic procedures using hematoxylin-eosin and resorcin fuchsin were applied to assess the general vessel wall architecture, that is, inflammation, vessel wall thickness, elastic lamellae, and cystic medial necrosis (focal loss of VSMC nuclei in the media). Expression of markers of differentiated VSMCs was investigated to determine differences in vessel wall maturation or differentiation between patients with BAV and TAV. Smooth muscle $22 \alpha$ (SM22 $\alpha$ ), smoothelin, and calponin were used as markers for fully differentiated contractile VSMCs ${ }^{18}$ and $\alpha$ smooth muscle actin ( $\alpha \mathrm{SMA})$ was used as a marker for differentiation of VSMCs and myofibroblasts. ${ }^{19}$ Lamin $\mathrm{A} / \mathrm{C}$, was investigated to explain possible differences between VSMC differentiation in BAV and TAV, because it has a pivotal role in the differentiation of myoblasts. ${ }^{20}$ Progerin, a splice variant of lamin $\mathrm{A} / \mathrm{C}$, was studied to further elucidate differences in the pathogenesis of aortopathy between the 2 valve types, because it has been suggested that progerin not only plays a role in Hutchinson-Gilford progeria syndrome but also in cardiovascular aging. ${ }^{21-23}$ We hypothesize that the BAV vessel wall has a maturation defect that underlies a more aggressive form of dilation.

\section{MATERIALS AND METHODS \\ Ethical Approval}

Sample collection and handling was carried out according to the official guidelines of the Medical Ethical Committee of Leiden University Medical Center (LUMC), Leiden, and the code of conduct of the Dutch Federation of Biomedical Scientific Societies (www.FMWV.nl). Six cryopreserved bicuspid human aortic valves were obtained from the Heart Valve Bank in Rotterdam (Erasmus University Medical Center, Rotterdam) originating from postmortem donors. These valves were declared unfit for implantation because of the bicuspid nature of the valves. The Advisory Board of the Heart Valve Bank allowed these valves to be included in the present project because the research was in line with the permission of the donation.

\section{Tissue Samples}

Samples of the ascending aorta were collected from individuals with BAV and TAV, with and without dilation. Material from patients with BAV without dilation was available in cases of stentless root replacement, the preferred technique for stentless valve implantation in LUMC. Dilation was clinically defined by reaching an ascending aortic wall diameter of 45 $\mathrm{mm}$ or more. ${ }^{24}$ The patients were divided into 4 groups: (1) TAV without dilation (TA, $\mathrm{n}=11$, mean age $64.5 \pm 9.0$ years) obtained post mortem; (2) TAV with dilation (TAD, $\mathrm{n}=12$, mean age $72.3 \pm 11.2$ years) collected during elective repair; (3) BAV without dilation (BA, $\mathrm{n}=17$, mean age $55.8 \pm 9.8$ years); (4) BAV with dilation (BAD, $\mathrm{n}=19$, mean age 60.7 \pm 7.8 years) obtained during elective repair. Patients with a proven genetic disorder (eg, Marfan disease) were excluded.

After excision, specimens were fixed in $4 \%$ formalin ( 24 hours), decalcified in a formic acid-formate buffer (120 hours), and embedded in paraffin. Transverse sections $(5 \mu \mathrm{m})$ were mounted on precoated Starfrost slides (Klinipath BV, Duiven, The Netherlands).

\section{Histologic Parameters}

Sections stained with hematoxylin-eosin and resorcin fuchsin were analyzed quantitatively for (1) periaortic inflammation (presence of a cellular infiltrate in the adventitia), indexed from 0 (no inflammatory cells), 2 (a few cells), 4 (groups of cells) to 6 (large clusters of cells); (2) maximum intimal thickness in micrometers (the distance between the endothelial layer and the first major internal elastic lamella, excluding atherosclerotic areas; (3) maximum medial thickness in micrometers (the distance between the first and last elastic lamella on the borderline with the adventitia. Furthermore, the organization of the elastic content was studied qualitatively. All specimens were reevaluated by an independent, experienced histopathologist who was blinded to the clinical data.

\section{Immunohistochemistry}

After deparaffinization, antigen retrieval was performed in a microwave oven in citrate buffer ( $\mathrm{pH} 6.0,12$ minutes), followed by treatment with $0.3 \% \mathrm{H}_{2} \mathrm{O}_{2}$ in phosphate buffered saline (PBS, pH 7.3, 20 minutes) to extinguish endogenous peroxidase activity. Subsequently, sections were rinsed briefly twice in PBS and once in PBS with $0.05 \%$ Tween-20 (PBS-T). Sections were incubated overnight at room temperature with the primary antibodies diluted in PBS-T and $1 \%$ bovine serum albumin (BSA, Sigma, St. Louis, Mo) (Table 1). Between the incubation steps, the slides were rinsed in PBS $(2 \times)$ and PBS-T $(1 \times)$. Bound antibodies were detected using 1-hour incubation with a secondary antibody diluted in PBS-T (Table 1). Subsequently, all slides, except for $\alpha$ SMA, were incubated with $\mathrm{ABC}$ reagent (Vector Laboratories, Burlingame, Calif; PK 6100) for 45 minutes. Control stainings were performed using PBS-T and BSA as the first incubation step. Slides were incubated with

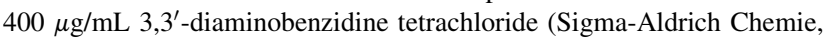
Buchs, Switzerland; D5637) dissolved in Tris-maleate buffer to which 20 $\mu \mathrm{L}$ of $\mathrm{H}_{2} \mathrm{O}_{2}$ were added ( $\mathrm{pH} 7.6,10$ minutes). After rinsing, counterstaining was performed with $0.1 \%$ hematoxylin (Merck, Darmstadt, Germany) (5 seconds), followed by rinsing in tap water (10 minutes). After dehydration, sections were mounted in Entellan (Merck, Darmstadt, Germany). Sections used for (semi)quantitative and morphometric analysis were stained in the same batch.

\section{Double Immunofluorescence Staining}

Deparaffinization, rehydration, and antigen retrieval were performed in the manner described earlier. Sections were incubated with the primary 
TABLE 1. Immunohistochemistry reagents

\begin{tabular}{|c|c|c|c|}
\hline Primary antibody & Vendor (order number) & Concentration & Secondary antibody \\
\hline Anti-lamin A/C & Chemicon, Billerica, Mass (MAB3211) & $1: 50$ & $\begin{array}{l}\text { HAM (1:200) and NHS (1:66) } \\
\text { (Vector Laboratories, Burlingame, Calif, BA-2000) } \\
\quad \text { (Brunschwig Chemie, Switzerland, S-2000) }\end{array}$ \\
\hline Anti-progerin & $\begin{array}{l}\text { Kindly provided by K. Djabali (Department of } \\
\text { Dermatology, Colombia University, New York, NY) }\end{array}$ & $1: 50$ & $\begin{array}{l}\text { GAR (1:200) and NGS (1:66) } \\
\text { (Vector Laboratories, Burlingame, Calif, BA-1000 and } \\
\text { S1000) }\end{array}$ \\
\hline Anti- $\alpha$ SMA & Sigma-Aldrich Chemie, Buchs, Switzerland (A2547) & $1: 5000$ & $\begin{array}{l}\text { RAM-PO }(1: 250) \\
(\mathrm{DAKO} \text { p } 0260)\end{array}$ \\
\hline Anti-SM $22 \alpha$ & Abcam, Cambridge, England (AB14106) & $1: 100$ & GAR and NGS \\
\hline Anti-human calponin & Sigma-Aldrich Chemie, Buchs, Switzerland (C2687) & $1: 6000$ & HAM and NHS \\
\hline Anti-smoothelin & Progen Biotechnik, Heidelberg, Germany (d4816101) & $1: 200$ & HAM and NHS \\
\hline
\end{tabular}

$H A M$, Horse-antimouse-biotin; $N H S$, normal horse serum; GAR, goat-antirabbit-biotin; $N G S$, normal goat serum; $R A M-P O$, peroxidase-conjugated rabbit antimouse.

antibodies, progerin and lamin $\mathrm{A} / \mathrm{C}$ (overnight, $4^{\circ} \mathrm{C}$ ) (Table 1). The secondary antibodies used were 1:500 Cy3 donkey antimouse IgG (Jackson Immunoresearch, Newmarket, England; 715-165-150) and 1:500 Alexa Fluor 647 donkey antirabbit IgG (Invitrogen, Grand Island, $\mathrm{NY}$; A-31573) (1 hour, $\left.20^{\circ} \mathrm{C}\right)$. The nuclei were counterstained for visualization with 4',6-diamidino-2-phenylindole (Sigma-Aldrich). All slides were mounted with ProlonGold (Invitrogen, P36930).

\section{Immunohistochemical and Morphometric Analyses}

Sections were studied with a Leica BM5000 microscope equipped with plan achromatic objectives (Leica Microsystems, Wetzlar, Germany). The cytoplasmatic level of expression of $\alpha \mathrm{SMA}, \mathrm{SM} 22 \alpha$, calponin, and smoothelin was indexed at 3 predetermined locations (left, middle, and right) of every tissue section, referred to as the microscopic field (MF), from 0 (no expression in VSMCs), 2 (expression in less than one-third of all VSMCs), 4 (expression in two-thirds of all VSMCs) to 6 (expression in all VSMCs). This was maintained for all stainings.

To determine the lamin-progerin balance, in each stained section the number of positively stained nuclei was counted using ImageJ in the 3 MFs. A threshold was applied to filter background noise. The total number of nuclei (positively stained and negative) was equal in all specimens. In each MF, the number of lamin- and progerin-positive nuclei was therefore normalized to the total number of nuclei per $100,000 \mu \mathrm{m}^{2}$. The number of normalized lamin- and progerin-positive nuclei was averaged between the 3 MFs.

\section{Western Blot}

Aortic tissue $(60 \mathrm{mg})$ was homogenized in $300 \mu \mathrm{L}$ of lysis buffer (Cell Signalling Technology, Beverly, Mass). Whole protein concentration in the cell lysate was measured using the BCA protein assay (Thermo Fisher Scientific, Rockford, Ill). Lamin A/C expression was quantified following procedures described previously, ${ }^{25}$ by using lamin $\mathrm{A} / \mathrm{C}$ rabbit polyclonal antibody (sc-20681) together with bovine antirabbit IgG-AP (sc-2372) secondary antibody (Santa Cruz Biotechnology, Santa Cruz, Calif) to detect lamin $\mathrm{A} / \mathrm{C}$ protein. Band intensity was analyzed using ImageJ software. To compare band intensity between different blots, an internal control sample (Rest) was run on every blot. Glyceraldehyde-3phosphate dehydrogenase (GAPDH) antibody (sc-47724; Santa Cruz Biotechnology) was used as loading control and with the calibration sample.

\section{Statistical Analysis}

All numerical data are presented as the mean \pm standard deviation of $3 \mathrm{MFs}$ on each stained slide. Statistical differences were evaluated with the Mann-Whitney $U$ test for comparison between the groups. SPSS 20.0 (SPSS Inc. Chicago, Ill) was used for the statistical analyses.
An additional 1-, 2- and 3-way analysis of variance test was performed to correct for age and gender and it was found that both factors were not confounding in this study. GraphPad software (GraphPad Software, Inc, San Diego, Calif) was used to create graphics of the statistical analyses.

\section{RESULTS \\ Histopathology}

The ascending aorta consists of a tunica intima, media, and adventitia, with endothelium lining the lumen (Figure $1, A, D, H$, and $K$ ). The border between the fine elastic fibers in the tunica intima and more regular thick elastic lamellae in the tunica media is delineated by an internal elastic lamella (Figure $1, B$ and $I$ ). The media to adventitia border is marked by the last elastic lamella of the media. The absolute intimal thickness was significantly smaller in BA compared with TA and BAD compared with TAD $(P<.001, P<.0001$, respectively) (Figure $1, B, I$, and $O$ ).

The media, however, was significantly thicker in all specimens in the BAV groups (BA and BAD) compared with the TAV groups (TA and TAD) $(P<.01$ and $P<.001$, respectively) (Figure $1, A, H$, and $P$ ). Differences in the organization of the elastic lamellae were also observed. In the TA group, the elastic lamellae had a dense regular distribution (Figure 1,C), whereas in the TAD group the lamellae appeared fragmented and the distance between the lamellae was enlarged (Figure 1,E). The BA group contained well-organized lamellae, even more neatly regulated compared with the TA group (Figure $1, J$ ). In the BAD group, the architecture of the elastic lamellae remained regular (Figure 1, $L$ ). Specimens from the BAD group also showed less cystic medial necrosis (focal loss of VSMC nuclei in the media) compared with specimen from the TAD group (Figure 1, $G$ and $N$ ). The adventitia in all specimens harbors fat cells, fibroblasts, nerve fibers, a few quiescent resident inflammatory cells, and vasa vasorum. The amount of adventitial fat cells (Figure 1, $M$ ) was not different between the 4 groups (data not shown). Periaortic inflammation was significantly more profound 

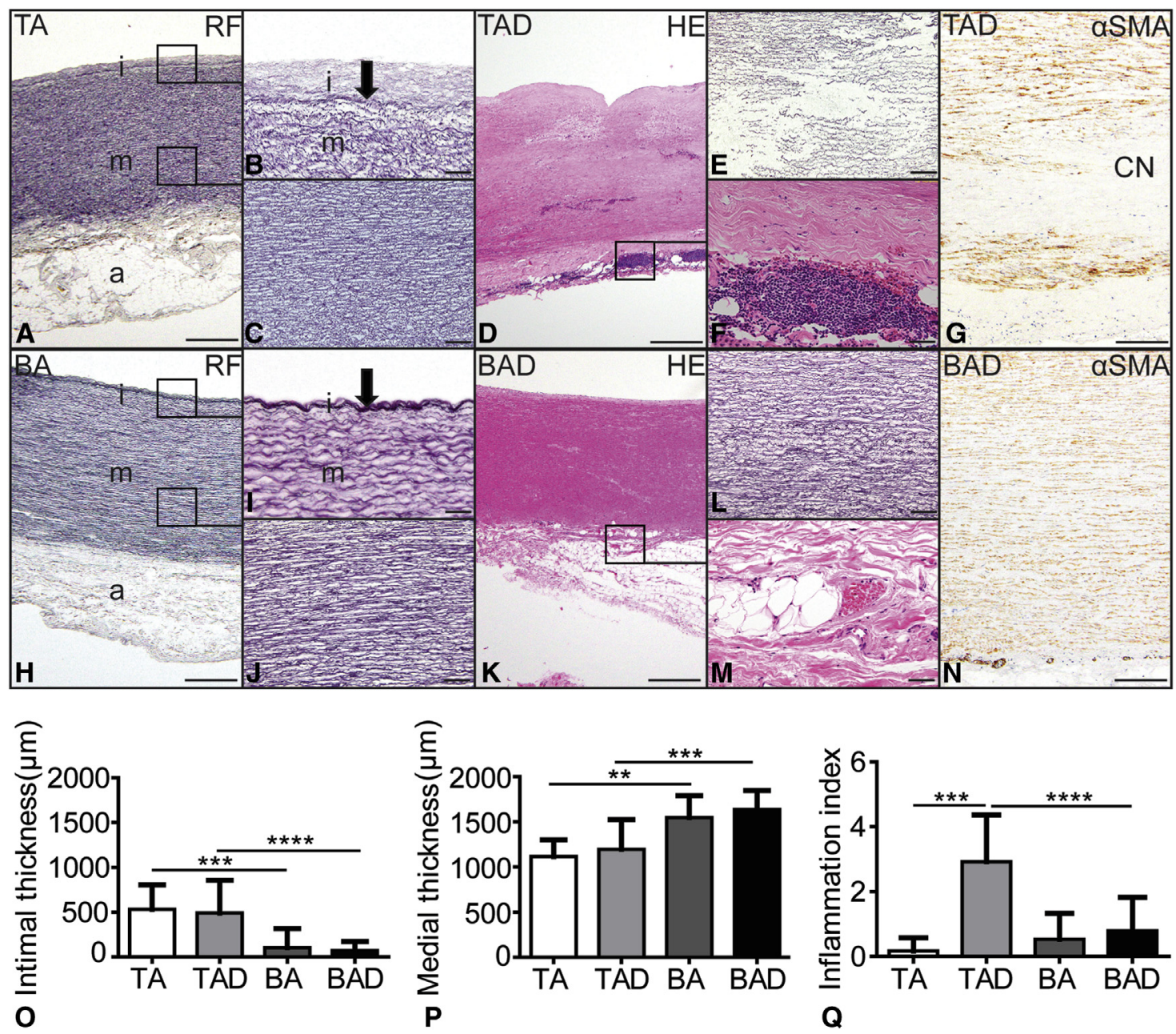

FIGURE 1. Transverse sections of the aortic wall (A-N). The aorta consists of an intima (i), media (m), and adventitia (a) indicated in A and H. The media and intima are separated by the internal elastic lamella (arrows in B and I). A very compact layering of medial elastic lamellae is seen in TA (C) compared with the somewhat more loosely organized yet regular lamellae in BA (J). The latter is maintained in the BAD group (L), in contrast to the TAD where the lamellae become fragmented and disorganized after dilation (E). Medial thickness is significantly increased in BA and BAD compared with TA and TAD (P). The reverse is seen for the intimal thickness, which is significantly thinner in $\mathrm{BA}$ and $\mathrm{BAD}(\mathrm{O}) . \alpha \mathrm{SMA}$ staining $(\mathrm{G}, \mathrm{N})$ exhibits more severe cystic medial necrosis $(C N)$ in TAD compared with BAD $(\mathrm{G})$. Periaortic inflammation (box in D, indexed as 6) is significantly more severe in TAD compared with BAD (D, F, K, M, and Q). $T A$, Tricuspid valve, without dilation; $T A D$, tricuspid valve, with dilation; $B A$, bicuspid valve, without dilation; $B A D$, bicuspid valve, with dilation; $R F$, resorcin fuchsin; $H E$, hematoxylin-eosin; $\alpha S M A, \alpha$ smooth muscle actin. Scale bars: A, D, G, H, K, and N, $500 \mu \mathrm{m}$; B, C, E, F, I, J, L, and M, $50 \mu \mathrm{m}$.

in the TAD group compared with the TA and BAD groups $(P<.001$ and $P<.0001$, respectively) (Figure $1, D, F, K$, $M$, and $Q$ ).

\section{Altered Pattern of Smooth Muscle Cell Expression in the Aorta of Patients With BAV Compared With TAV}

Differences in the expression of actin-positive VSMCs were noted between the groups. The ascending aorta from BAV showed less $\alpha$ SMA expressing VSMCs compared with TAV, in both dilated and nondilated specimens $(P<.05)$ (Figure 2, $A, B$, and $C$ ). Less expression in BAV was not due to focal vessel wall degeneration, rather individual cells expressed less $\alpha$ SMA. The results obtained for $\alpha$ SMA were mirrored by the expression pattern of
$\operatorname{SM} 22 \alpha(P<.01)$ (Figure 2, $D, E$, and $F$ ) and calponin (data not shown). The intima of the aortic wall stained positive for SM22 $\alpha$, but not for $\alpha$ SMA (Figure 2, $A, B, D$, and $E$ ). Smoothelin expression in the TA group was seen mainly in the outer media compared with the middle and inner media, with a significant degenerative loss of expression in the middle media of the TAD group $(P<.001)$ (Figure 2, $G, J$, and $I)$. Smoothelin expression in the BA and BAD groups was nearly absent $(P<.0001$ and $P<.001$, respectively) (Figure $2, H, K$, and $I$ ).

\section{Lamin A/C and Progerin Expression}

The expression of the nuclear protein lamin $\mathrm{A} / \mathrm{C}$ was seen in the intima and media. Significantly less lamin 


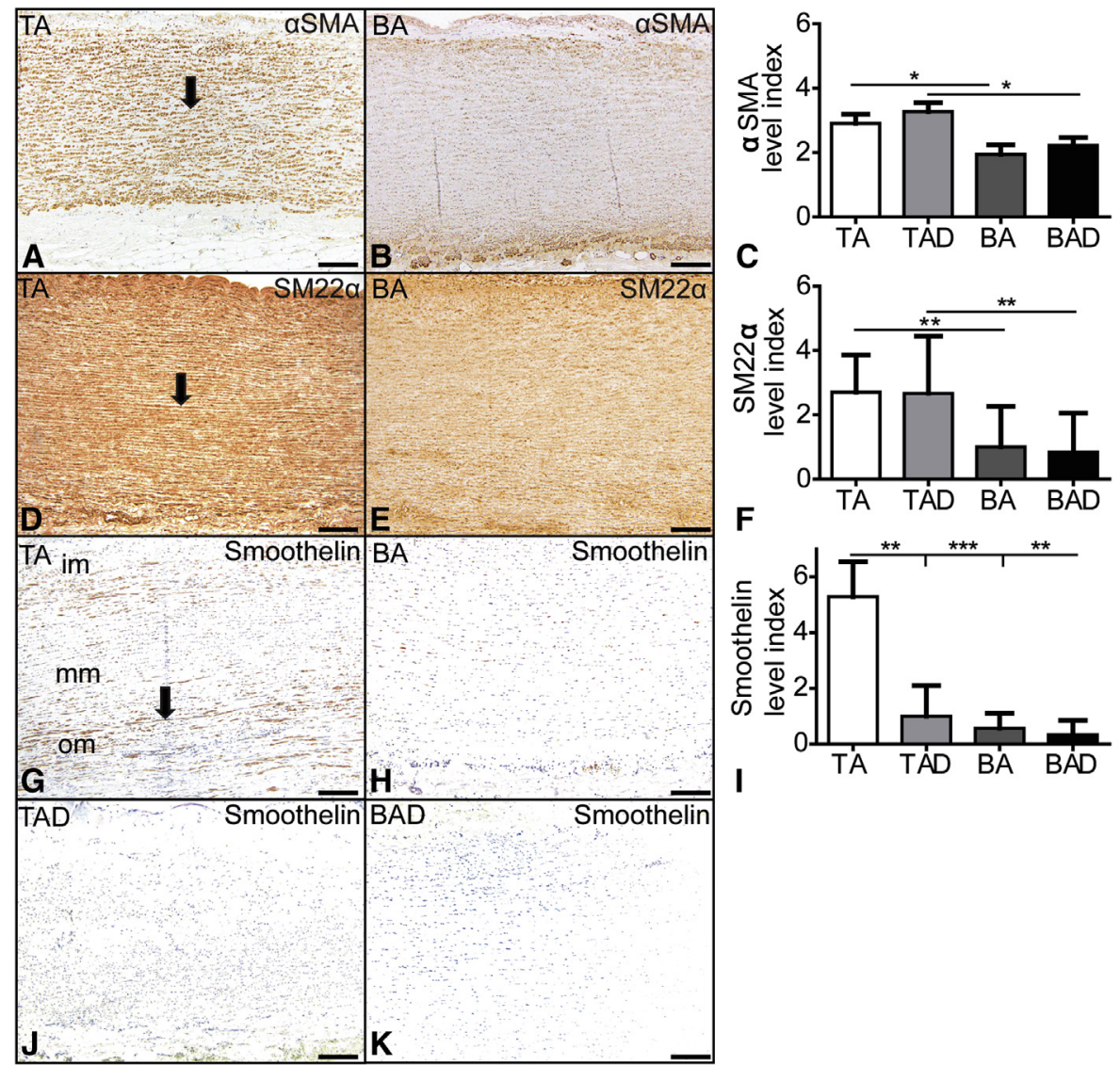

FIGURE 2. Transverse sections of the aortic wall (A, B, D, E, G, H, J, K). Cytoplasmatic 3,3'-diaminobenzidine tetrachloride vascular smooth muscle cell (VSMC) staining, with positivity depicted by an arrow in A, D, and G. The number of $\alpha$ SMA expressing VSMCs is significantly lower in BA (B, indexed as 0 ) than TA (A, indexed as 6), which is maintained after dilation (BAD and TAD, respectively) (C). The number of SM22 $\alpha$ expressing VSMCs is also significantly lower in BA (E, indexed as 2) than TA (D, indexed as 6) and in BAD compared with TAD (F). VSMCs expressing smoothelin were only seen in the TA, mainly in the outer (om) compared with the middle ( $\mathrm{mm}$ ) and inner media (im) (G, indexed as 4) and nearly absent in the other groups $(\mathrm{H}, \mathrm{J}$, and $\mathrm{K}$, indexed as 0 ) (I). TA, Tricuspid valve, without dilation; $T A D$, tricuspid valve, with dilation; $B A$, bicuspid valve, without dilation; $B A D$, bicuspid valve, with dilation; $\alpha S M A, \alpha$ smooth muscle cell actin; SM22 $\alpha$, smooth muscle cell $22 \alpha$. Scale bars: $200 \mu \mathrm{m}$.

A/C-positive nuclei were observed in the BA group compared with the TA group $(P<.01)$ (Figure $3, A, E$, and $G$ ). We also investigated the expression of progerin. Confocal microscopy revealed progerin expression at the nuclear lamina (Figure 3, $C$ and $F$ ), whereas lamin A/C was also distributed in the nucleoplasm (Figure $3, B$ and $D$ ), confirming that the 2 antibodies recognize different proteins. Morphologic analyses of progerin expression patterns revealed a significant increase in the TAD group compared with the TA group $(P<.05)$ (Figure $3, H)$, whereas no such increase was seen between the BAD and BA groups. Moreover, the expression in the BAD group was significantly lower than the in TAD group $(P<.05)$ (Figure $3, H)$.

Lamin $\mathrm{A} / \mathrm{C}$ expression in the $\mathrm{BA}$ and $\mathrm{TA}$ groups was quantified by performing a Western blot. The level of GAPDH was used as calibration of the samples. The results of lamin A/C quantification showed the same trend as the immunohistochemistry data (Figure 4).

\section{DISCUSSION}

Previous studies demonstrated that patients with BAV have more severe aortic wall abnormalities than patients with TAV. ${ }^{14}$ The results of our study provide evidence that the aortic wall in BAV is intrinsically different and presents with a maturation defect compared with the aortic wall in TAV. This maturation defect is maintained in the dilated aortic wall, which also has a different basic architecture in BAV compared with TAV. The significant difference observed remained after correcting for age and gender.

Most literature data refer to comparison of the dilated wall in patients with TAV and BAV. ${ }^{11,12,14}$ We had the opportunity to compare the wall in 4 groups, including a unique nondilated BA population, which allowed us to define its basic structural differences. Our observation that the inflammatory component is minimal in BAD compared with TAD, despite the presence of a similar degrees of aortic dilation, confirms the findings of 

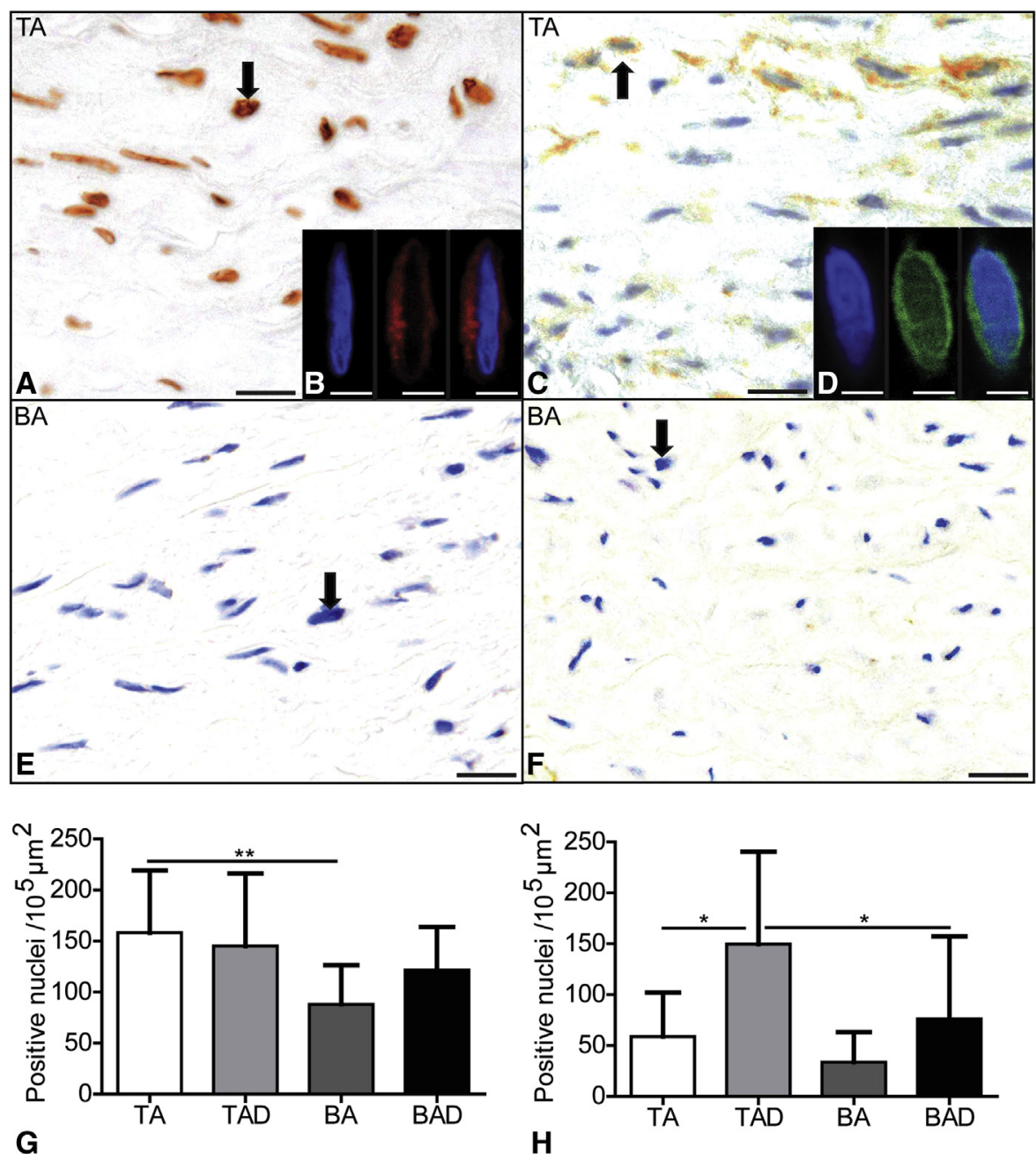

FIGURE 3. Transverse histologic sections of the aortic wall (A-F). Nuclear 3,3'-diaminobenzidine tetrachloride (DAB) staining of lamin A/C (A, brown, see arrow; E, absent staining, see arrow). The number of lamin A/C-expressing nuclei is significantly lower in the BA group compared with the TA group (G). Nuclear DAB staining of progerin is shown in $\mathrm{C}$ (brown, see arrow) and $\mathrm{F}$ (absent staining, see arrow). The number of progerin-expressing nuclei significantly increases with wall dilation in TAD $(\mathrm{H})$, which is not seen in BAD. The number of progerin-expressing nuclei is significantly lower in BAD compared with TAD $(\mathrm{H})$. Confocal images of consecutive sections (B and D). Nuclei are counterstained with diamidino phenylindole (B and D, blue). Immunofluorescence shows nuclear distribution of lamin A/C (red, B) and nuclear envelope localization of progerin (green, D). TA, Tricuspid valve, without dilation; TAD, tricuspid valve, with dilation; $B A$, bicuspid valve, without dilation; $B A D$, bicuspid valve, with dilation. Scale bars: A, C, E, and F, $20 \mu \mathrm{m}$; B and D, $1 \mu \mathrm{m}$.

Matthias Bechtel and colleagues. ${ }^{26}$ The significantly thinner intima in all our BAV specimens compared with the TAV specimens is a novel finding and might explain the less frequent observation of atherosclerosis in the BAV aortic wall. ${ }^{26}$ The aortic media appeared significantly thicker in all patients with BAV compared with TAV, which was not found earlier. ${ }^{12}$ Specimens in the BA and BAD groups showed relatively well-organized, albeit thinner, elastic lamellae, with a larger interlamellar distance compared with those from patients with TAV, a finding previously described for the BAD group, but not for the nondilated BA group. ${ }^{11,12,14}$

We propose that the finer elastic lamellae observed combined with an increase in extracellular matrix in the nondilated BA group might add to the increased susceptibility for dilation of the media as has been suggested in literature. ${ }^{27}$

VSMCs have the ability to undergo a phenotypic switch from a quiescent contractile state to a less mature, proliferative synthetic state. Disability of this switch has been shown to play a critical role in a variety of cardiovascular diseases. ${ }^{18,28}$ By using differentiation markers of contractile VSMCs, we showed less maturation in patients with BAV compared with TAV in both dilated and nondilated specimens. This was particularly prominent for smoothelin, a specific marker of highly differentiated VSMCs, which was significantly less in the TAD group compared with the TA group, and, as we show for the first 


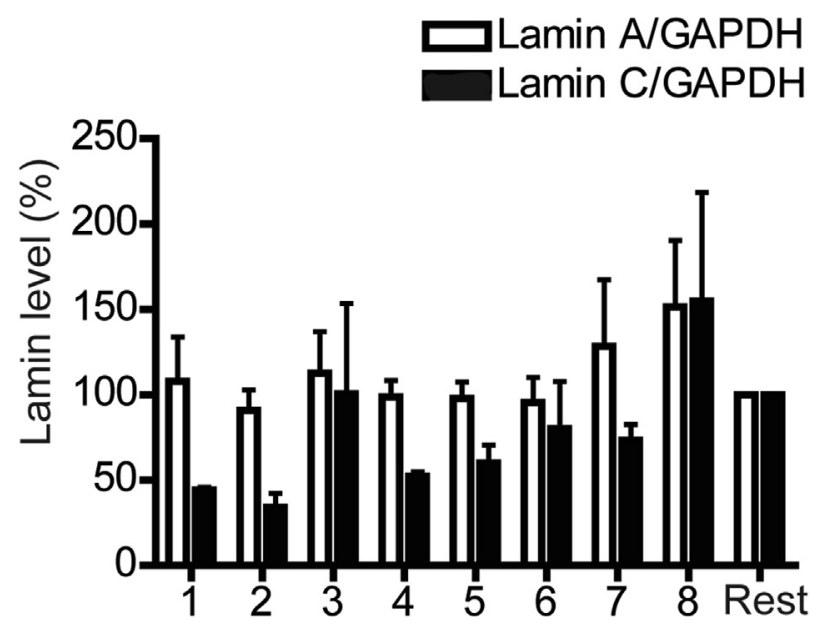

FIGURE 4. Western blot of cell lysate from 8 samples of nondilated aortic wall with a bicuspid aortic valve (BA, 1-4) and tricuspid valve (TA, 5-8) and glyceraldehyde-3-phosphate dehydrogenase (GAPDH) used as an internal control sample (Rest). The lamin A/C protein level in each sample was determined by densitometry, performed using ImageJ software, and normalized. The band intensity of the Rest was used as calibrator. The Coomassie Blue stained gel showed equal loading of proteins with a molecular mass of $69 \mathrm{kDa}$ (lamin A), and $62 \mathrm{kDa}$ (lamin C). Bar diagram in white, lamin $\mathrm{A}$ level in \%; and in black, lamin $\mathrm{C}$ level in \%.

time, nearly absent in all BAVs. These findings correlate with the structurally less well-differentiated BA wall compared with the normal TA wall, which could explain aortic wall vulnerability in bicuspidy.

This hypothesis is further supported by the novel finding that lamin $\mathrm{A} / \mathrm{C}$ is significantly less expressed in all patients with BAV. The nuclear intermediate filament lamin A and C proteins are expressed from the same LMNA gene by alternative splicing. Lamin $\mathrm{A} / \mathrm{C}$ are of utmost importance in regulating transcription, organizing the chromatin, and protecting the nucleus from mechanical stress during cell development. ${ }^{29}$ The role of lamin A/C in normal VSMC differentiation has not been reported so far, however mice with reduced expression show nuclear deformation and impaired transcription response to mechanical stress. ${ }^{30}$ Low expression and an altered lamin $\mathrm{A} / \mathrm{C}$ ratio in the $\mathrm{BA}$ group, which is maintained in the BAD group, suggest that the lamins might also play a role in VSMC differentiation and maturation, and thereby affect the aortic dilation process. In line with these data is the remarkably increased progerin expression in the degenerated TAD group, which can be linked to normal cardiovascular aging. ${ }^{21-23}$ The relative lack of progerin in the BA group and the nonsignificant increase subsequently in the BAD group indicate that in the immature wall of patients with $\mathrm{BAV}$, the degenerative changes are of a different nature compared with TAV and do not involve so much accelerated aging, emphasizing that BAV and TAV are clearly different entities. Although, in general, reduced levels of lamin $\mathrm{A} / \mathrm{C}$ and associated proteins affect the transcription of genes ${ }^{31}$ by, for example, histone modifications, ${ }^{32}$ it is of utmost importance to understand its role in cell type-specific differentiation and dedifferentiation processes. Future studies should focus on the regulation of lamin A and C in VSMCs and the role of these lamins in vessel wall pathology.

In conclusion, our data show that the aortic wall in patients with BAV is intrinsically different from patients with TAV, in whom inflammation and accelerated aging lead to aortic pathology. A maturation defect of the aortic wall in BAV, showing less well-differentiated VSMCs and low lamin $\mathrm{A} / \mathrm{C}$ expression, might be the cause of the increased risk of aortic wall dilation. We have thereby shown with the amended markers that there is clearly a different substrate for aortopathy, seen more frequently and at a younger age, in BAV compared with TAV. As the nondilated aortic wall has already been found to be different, there is a need for reevaluation of the role of hemodynamics as a possible primary cause for the dilation in BAV. Future experimental research should focus on whether pharmacologic interventions can influence maturation in the aortic wall, which would be valuable in limiting clinical disease progression in the immature BAV wall.

\section{Study Limitations}

A limitation of our study is that we did not have aortic wall specimens from control patients (children and adolescents) to indicate whether the observed immaturity in BAV is the result of a maturation defect or a matter of nonprogression of normal maturation. This can only be studied in the various existing BAV mouse models.

The authors thank the Heart Valve Bank Rotterdam, The Netherlands, for providing the cryopreserved aortic tissues.

\section{References}

1. Siu SC, Silversides CK. Bicuspid aortic valve disease. J Am Coll Cardiol. 2010; 55:2789-800.

2. Fedak PW, David TE, Borger M, Verma S, Butany J, Weisel RD. Bicuspid aortic valve disease: recent insights in pathophysiology and treatment. Expert Rev Cardiovasc Ther. 2005;3:295-308.

3. Ferencik M, Pape LA. Changes in size of ascending aorta and aortic valve function with time in patients with congenitally bicuspid aortic valves. Am J Cardiol. 2003;92:43-6.

4. Beroukhim RS, Kruzick TL, Taylor AL, Gao D, Yetman AT. Progression of aortic dilation in children with a functionally normal bicuspid aortic valve. Am J Cardiol. 2006;98:828-30.

5. Girdauskas E, Borger MA, Secknus MA, Girdauskas G, Kuntze T. Is aortopathy in bicuspid aortic valve disease a congenital defect or a result of abnormal hemodynamics? A critical reappraisal of a one-sided argument. Eur J Cardiothorac Surg. 2011;39:809-14.

6. Braverman AC, Guven H, Beardslee MA, Makan M, Kates AM, Moon MR. The bicuspid aortic valve. Curr Probl Cardiol. 2005;30:470-522.

7. Yasuda H, Nakatani S, Stugaard M, Tsujita-Kuroda Y, Bando K, Kobayashi J, et al. Failure to prevent progressive dilation of ascending aorta by aortic valve replacement in patients with bicuspid aortic valve: comparison with tricuspid aortic valve. Circulation. 2003;108(Suppl 1):II291-4. 
8. Ikonomidis JS, Jones JA, Barbour JR, Stroud RE, Clark LL, Kaplan BS, et al. Expression of matrix metalloproteinases and endogenous inhibitors within ascending aortic aneurysms of patients with bicuspid or tricuspid aortic valves. J Thorac Cardiovasc Surg. 2007;133:1028-36.

9. Boyum J, Fellinger EK, Schmoker JD, Trombley L, McPartland K, Ittleman FP, et al. Matrix metalloproteinase activity in thoracic aortic aneurysms associated with bicuspid and tricuspid aortic valves. J Thorac Cardiovasc Surg. 2004; 127:686-91.

10. Koullias GJ, Korkolis DP, Ravichandran P, Psyrri A, Hatzaras I, Elefteriades JA. Tissue microarray detection of matrix metalloproteinases, in diseased tricuspid and bicuspid aortic valves with or without pathology of the ascending aorta. Eur J Cardiothorac Surg. 2004;26:1098-103.

11. Niwa K, Perloff JK, Bhuta SM, Laks H, Drinkwater DC, Child JS, et al. Structural abnormalities of great arterial walls in congenital heart disease: light and electron microscopic analyses. Circulation. 2001;103:393-400.

12. Bauer M, Pasic M, Meyer R, Goetze N, Bauer U, Siniawski H, et al. Morphometric analysis of aortic media in patients with bicuspid and tricuspid aortic valve. Ann Thorac Surg. 2002;74:58-62.

13. Nataatmadja M, West M, West J, Summers K, Walker P, Nagata M, et al. Abnormal extracellular matrix protein transport associated with increased apoptosis of vascular smooth muscle cells in Marfan syndrome and bicuspid aortic valve thoracic aortic aneurysm. Circulation. 2003;108(Suppl 1): II329-34.

14. de Sa M, Moshkovitz Y, Butany J, David TE. Histologic abnormalities of the ascending aorta and pulmonary trunk in patients with bicuspid aortic valve disease: clinical relevance to the Ross procedure. J Thorac Cardiovasc Surg. 1999;118:588-94.

15. Della CA, Quarto C, Bancone C, Castaldo C, Di MF, Nurzynska D, et al. Spatiotemporal patterns of smooth muscle cell changes in ascending aortic dilatation with bicuspid and tricuspid aortic valve stenosis: focus on cell-matrix signaling. J Thorac Cardiovasc Surg. 2008;135:8-18. 18.e1-2.

16. Phillippi JA, Green BR, Eskay MA, Kotlarczyk MP, Hill MR, Robertson AM, et al. Mechanism of aortic medial matrix remodeling is distinct in patients with bicuspid aortic valve. J Thorac Cardiovasc Surg. 2013; http://dx.doi.org/ 10.1016/j.jtcvs.2013.04.028.

17. Fedak PW, de Sa MP, Verma S, Nili N, Kazemian P, Butany J, et al. Vascular matrix remodeling in patients with bicuspid aortic valve malformations: implications for aortic dilatation. J Thorac Cardiovasc Surg. 2003;126: 797-806.

18. Owens GK, Kumar MS, Wamhoff BR. Molecular regulation of vascular smooth muscle cell differentiation in development and disease. Physiol Rev. 2004;84: 767-801.

19. Leslie KO, Taatjes DJ, Schwarz J, vonTurkovich M, Low RB. Cardiac myofibroblasts express alpha smooth muscle actin during right ventricular pressure overload in the rabbit. Am J Pathol. 1991;139:207-16.
20. Frock RL, Kudlow BA, Evans AM, Jameson SA, Hauschka SD, Kennedy BK Lamin $\mathrm{A} / \mathrm{C}$ and emerin are critical for skeletal muscle satellite cell differentiation. Genes Dev. 2006;20:486-500.

21. Olive M, Harten I, Mitchell R, Beers JK, Djabali K, Cao K, et al. Cardiovascular pathology in Hutchinson-Gilford progeria: correlation with the vascular pathology of aging. Arterioscler Thromb Vasc Biol. 2010;30:2301-9.

22. Ragnauth CD, Warren DT, Liu Y, McNair R, Tajsic T, Figg N, et al. Prelamin A acts to accelerate smooth muscle cell senescence and is a novel biomarker of human vascular aging. Circulation. 2010;121:2200-10.

23. Bokenkamp R, Raz V, Venema A, DeRuiter MC, van MC, Olive M, et al. Differential temporal and spatial progerin expression during closure of the ductus arteriosus in neonates. PLoS One. 2011;6:e23975.

24. Hiratzka LF, Bakris GL, Beckman JA, Bersin RM, Carr VF, Casey DE Jr, et al. 2010 ACCF/AHA/AATS/ACR/ASA/SCA/SCAI/SIR/STS/SVM guidelines for the diagnosis and management of patients with thoracic aortic disease: executive summary. A report of the American College of Cardiology Foundation/American Heart Association Task Force on Practice Guidelines, American Association for Thoracic Surgery, American College of Radiology, American Stroke Association, Society of Cardiovascular Anesthesiologists, Society for Cardiovascular Angiography and Interventions, Society of Interventional Radiology, Society of Thoracic Surgeons, and Society for Vascular Medicine. Catheter Cardiovasc Interv. 2010;76:E43-86.

25. Mohamed SA, Radtke A, Saraei R, Bullerdiek J, Sorani H, Nimzyk R, et al. Locally different endothelial nitric oxide synthase protein levels in ascending aortic aneurysms of bicuspid and tricuspid aortic valve. Cardiol Res Pract. 2012;2012:165957.

26. Matthias Bechtel JF, Noack F, Sayk F, Erasmi AW, Bartels C, Sievers HH. Histopathological grading of ascending aortic aneurysm: comparison of patients with bicuspid versus tricuspid aortic valve. J Heart Valve Dis. 2003;12:54-9.

27. Shen YH, Hu X, Zou S, Wu D, Coselli JS, LeMaire SA. Stem cells in thoracic aortic aneurysms and dissections: potential contributors to aortic repair. Ann Thorac Surg. 2012;93:1524-33.

28. Fisher SA. Vascular smooth muscle phenotypic diversity and function. Physiol Genomics. 2010;42A:169-87.

29. Zuela N, Bar DZ, Gruenbaum Y. Lamins in development, tissue maintenance and stress. EMBO Rep. 2012;13:1070-8.

30. Lammerding J, Schulze PC, Takahashi T, Kozlov S, Sullivan T, Kamm RD, et al. Lamin A/C deficiency causes defective nuclear mechanics and mechanotransduction. J Clin Invest. 2004;113:370-8.

31. Spann TP, Goldman AE, Wang C, Huang S, Goldman RD. Alteration of nuclear lamin organization inhibits RNA polymerase II-dependent transcription. J Cell Biol. 2002; 156:603-8.

32. Galiova G, Bartova E, Raska I, Krejci J, Kozubek S. Chromatin changes induced by lamin $\mathrm{A} / \mathrm{C}$ deficiency and the histone deacetylase inhibitor trichostatin A. Eur J Cell Biol. 2008;87:291-303. 